\title{
Erratum to: Vector Potential of Electromagnetic Wave above Strongly Inductive Two-Layer Earth Surface
}

\author{
V. K. Balkhanov ${ }^{a, *}$, Yu. B. Bashkuev ${ }^{a}$, and L. Kh. Angarkhaeva ${ }^{a}$ \\ ${ }^{a}$ Institute of Physical Materials Science, Siberian Branch, Russian Academy of Sciences, \\ Ulan-Ude, Buryat Republic, 670047 Russia \\ *e-mail: ludang@rambler.ru
}

Received May 17, 2021; revised May 17, 2021; accepted May 17, 2021

DOI: $10.1134 / \mathrm{S} 106378422108020 \mathrm{X}$

Formula (18) should read as follows:

$$
D=\frac{\pi J_{0}}{\mu_{0}}\left[\left(\Omega_{10}+1\right)-\left(\Omega_{10}-1\right) \frac{L_{-}}{L_{+}}\right] \exp \left(-\mu_{0} l\right) .
$$

Formula (19) should read as follows:

$$
E=\frac{\pi J_{0}}{\mu_{0}}\left[\left(\Omega_{10}+1\right) \frac{L_{-}}{L_{+}}-\left(\Omega_{10}-1\right)\right] \exp \left(-\mu_{0} l\right) .
$$

Formula (20) should read as follows:

$$
\begin{gathered}
F=\frac{\pi J_{0}}{\mu_{0}}\left\{\left[\left(\Omega_{10}+1\right)-\left(\Omega_{10}-1\right) \frac{L_{-}}{L_{+}}\right] \exp \left(-\mu_{1} l\right)\right. \\
\left.+\left[\left(\Omega_{10}+1\right) \frac{L_{-}}{L_{+}}-\left(\Omega_{10}-1\right)\right] \exp \left(\mu_{1} l\right)\right\} \exp \left(-\mu_{0} l+\mu_{2} h\right) .
\end{gathered}
$$

The original article can be found online at https://doi.org/10.1134/S1063784219090032 